\title{
The Effects of Negative Values of Apparent Resistivity in TEM Surveys
}

\section{Cassiano Antonio Bortolozo ${ }^{1}$, Julian David Realpe Campaña1, Marco Antonio Couto Junior ${ }^{1,2}$, Jorge Luis Porsani1 ${ }^{1}$, Fernando Acácio Monteiro dos Santos ${ }^{3}$}

${ }^{1}$ Departamento de Geofísica, Instituto de Astronomia, Geofísica e Ciências Atmosféricas (IAG), Universidade de São Paulo (USP), São Paulo, Brasil

${ }^{2}$ Brazilian Geological Survey (CPRM), Belo Horizonte, Brazil

${ }^{3}$ University of Lisboa, Don Luiz Institute (IDL), Lisbon, Portugal

Email: cassiano@iag.usp.br, jdrealpec@gmail.com, marco.couto@cprm.gov.br, porsani@iag.usp.br, fasantos@fc.ul.pt

How to cite this paper: Bortolozo, C.A., Campaña, J.D.R., Junior, M.A.C., Porsani, J.L. and dos Santos, F.A.M. (2016) The Effects of Negative Values of Apparent Resistivity in TEM Surveys. International Journal o1 Geosciences, 7, 1182-1190. http://dx.doi.org/10.4236/ijg.2016.710088

Received: September 16, 2016

Accepted: October 24, 2016

Published: October 27, 2016

Copyright $\odot 2016$ by authors and Scientific Research Publishing Inc. This work is licensed under the Creative Commons Attribution International License (CC BY 4.0).

http://creativecommons.org/licenses/by/4.0/

\begin{abstract}
The calculation of apparent resistivity in electrical methods is relatively simple and consolidated in the literature. Furthermore, in the case of DC methods, the apparent resistivity values are for the most part, intuitive and enable an initial interpretation of the results. On the other hand, in TEM method the apparent resistivity values are not very intuitive and interpretation based only on the apparent resistivity values cannot be done reasonably. In this way, this paper presents a discussion about the reasons and effects of the negatives values of apparent resistivity in TEM soundings. The main objective of this paper is to clarify the meanings of these negatives values and the possible effects in TEM inversion.
\end{abstract}

\section{Keywords}

Transient Electromagnetic (TEM), Apparent Resistivity, Negative Values

\section{Introduction}

The concept of apparent resistivity is widely used in electrical and electromagnetic geophysical methods. This concept originates in the sense that the calculations of the electrical resistivity measured by these methods assume a homogeneous medium as a reference. But, once the geological substratum mostly presents heterogeneity, the measured resistivity is usually a weighted average of all geological structures detected. Thus, in geophysics, the term apparent resistivity normally is used for the data acquired in field (surface or in boreholes). In the case of electrical methods, the calculation of the apparent resistivity ends up being simpler compared to electromagnetic methods. 
Another advantage when working with apparent resistivity in electrical methods is that the values obtained are intuitive and allow some types of interpretations directly.

However, in electromagnetic methods, particularly in TEM method, the calculation of apparent resistivity is eventually more complex when compared to DC methods. The apparent resistivity values obtained in this case do not provide much information directly, since it does not have a very intuitive response for models with relative thin layers, as in the cases with sedimentary aquifers. Thus, it is not possible to obtain a reliable a priori interpretation based only on the apparent resistivity values of TEM. The calculations of apparent resistivity for the TEM method have many approximations [1]-[5] including different approaches to the early and late times [3]. Some works that seek a unique form of calculation, for both early and late times, there are already available [6] [7], but this kind of calculation is not widespread and is still not widely used in direct and inverse calculation software.

A more complex situation in relation to the calculation of apparent resistivity of the TEM method occurs with the use of data measured inside and outside of the transmitter loop in $1 \mathrm{D}$ and $2 \mathrm{D}$ surveys. With the formulations currently used to calculate the apparent resistivity responses, negative values of apparent resistivity are obtained outside the transmitter loop. Since negative electrical resistivity values have no physical meaning this problem is due to inaccurate calculation of apparent resistivity. The consequences associated with such negative values in the inversion of $1 \mathrm{D}$ and $2 \mathrm{D}$ data are discussed in the works of Campaña [8] and Bortolozo [9] respectively. The problems encountered are related to low resolution by these values, increased ambiguity and reduced stability of inversion processes. This article aims to discuss the reasons and effects of these negative values in TEM surveys. With the main objective clarifies the meaning of these values and the effects on TEM inversion.

\section{Apparent Resistivity}

The calculation of the subsurface resistivity assumes a homogeneous and isotropic medium, but the subsurface in a real case is not homogeneous and the targets of geophysical surveys are often the variations in the distribution of the substratum resistivity. The term apparent resistivity $\left(\rho_{a}\right)$ comes from the fact that the voltage measured in the receiver coil is related to the passage of the secondary current (eddy currents) induced in a heterogeneous environment. The current flow passes through several underground geoelectrical layers with different resistivities. The measured difference of electrical potential is not due to the current flow passing through a single medium with resistivity $\rho$, but is equivalent to the current passing through a medium with resistivity $\rho_{a}$. Wherein the resulting resistivity is equivalent to a weighted average of all strata which the current passed through. Therefore, the apparent resistivity term is used because the obtained resistivity is not directly linked to a homogeneous medium, but the set of structures and lithology in the path of the induced current.

In the case of TEM, the sampled area will be related to the volume involved by the smoke rings (Figure 1) of the eddy currents in the underground. These currents will 
transit like rings in the subsurface and the apparent resistivity corresponds to the average resistivity of underground geology. The lines of secondary current will be distorted similarly to the situation shown in Figure 1. Furthermore, the TEM survey seeks to explore this distortion effect in the current flow on the substrate. With the measurement of voltages in the receiving coil at increasingly later times allows reaching deeper layers of the substrate or exploring the lateral variations of resistivity.

\section{Apparent Resistivity Inside and Outside of the Transmitter Loop}

In the case of $1 \mathrm{D}$ and $2 \mathrm{D}$ TEM surveys, it is common to use soundings outside of the current loop for the analysis of the secondary magnetic field [10]. However, the analysis and inversion of these data in the form of apparent resistivity becomes compromised by the fact that the calculation formulas usually take into account only the soundings within the transmitter loop. In the 1D case, an example of calculation of apparent resistivity is given in Nielsen and Baumgartner [4] as:

$$
\rho_{a}=\left[\frac{\sqrt{\pi} a^{2} n b^{2}}{20(\partial B / \partial t)}\right]^{2 / 3}+\left(\frac{\mu_{0}}{t}\right)^{5 / 3}
$$

where $b$ is the radius of the receiving loop and $n$ the number of turns, $a$ is the radius of the transmitter loop, $t$ the time and $\mu_{0}$ the magnetic permeability of free space. In the $1 \mathrm{D}$ case, the calculation of $\partial B / \partial t$ is made only to the center of the transmitter loop (central loop array).

For the 2D case, as shown in the work of Oristaglio and Hohmann [2], the 2D apparent resistivity $\left(\rho_{a}\right)$ is given in the form of:

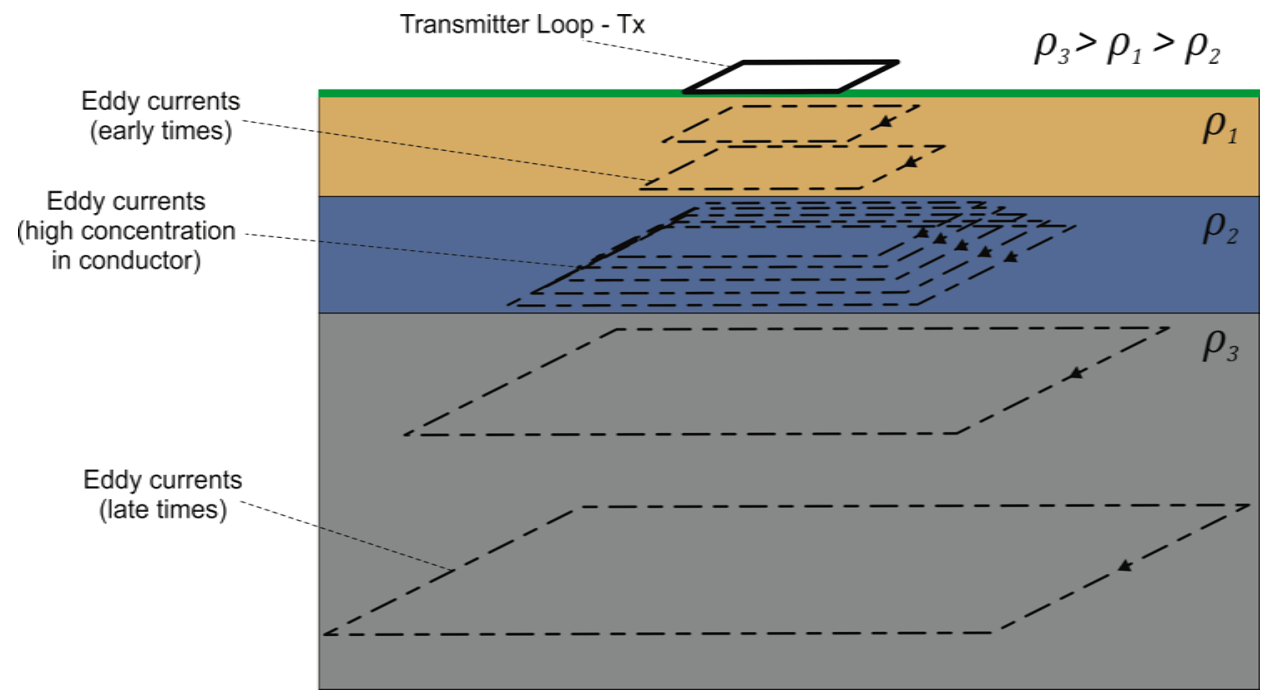

Figure 1. The schematic figure represents a model with three layers with different resistivities, and the intermediate layer has a lower resistivity. The dashed lines represent the lines of the secondary currents in the form of "smoke rings", and how they are distorted by the resistivity variation. 


$$
\rho_{a}=\frac{\mu_{0}^{2} L_{f}}{16 \pi t^{2} \partial B / \partial t}
$$

in which, $L_{f}$ is the distance between the wires of the transmitter loop. Equation (2) assumes the source as two infinite wires and the location of the receiver coil inside the both wires.

In the $1 \mathrm{D}$ case, the formula of apparent resistivity (Equation (2)) is designed for measurements made inside the loop. It is assumed the basic premise that the lines of the $B$ field are positive out of the ground into the air. When measures are taken only within the transmitter loop, the premise to calculate the apparent resistivity is not violated and calculations are carried out correctly. But, when the receiving coil is positioned outside the transmitter loop, in the case of fixed-loop array for instance, the premise of positive $B$ field is violated. Then, negative values of apparent resistivity are obtained in the form presented in the work Campaña [8].

The reason for this, as already discussed, is because the secondary field is generated by currents in subsurface in the form of "smoke rings". The shape of the "smoke rings" may be defined as projections of the transmitter loop in subsurface with areas that gradually increase with depth. We can therefore understand the "smoke rings" for each measuring time, as a current loop transmitter with an area that varies over time, with increasingly larger size and depth. Obviously, the current intensity decreases with time, but for understanding how the behavior of the "smoke rings" affects the calculation of apparent resistivity is not needed to take it into account.

In Figure 2 is shown a schematic of how the secondary current behaves when compared to the primary current. In Figure 2(a) shows the transmitter loop when the primary current passes through it and the magnetic field $B$ associated with it. In Figure 2 (b) is presented the same array in a given later instant time with the secondary currents in the subsurface with its associated magnetic field $B_{S}$. The current pattern is in the form of "smoke rings", i.e., they are increasing projections of the transmitter loop with larger areas. The following text will reference to this pattern of secondary current in a given time, in the form of a greater projection of the transmitter loop as "secondary current loop". This reference will serve to facilitate the understanding of the geometry of currents and is not a usual nomenclature for this type of induced secondary current.

To calculate the apparent resistivity is assumed a positive $B$ field, so that in this way, the apparent resistivity values are calculated consistently. Once the "smoke rings" are always greater projections of the transmitter loop, we can assume that the "minor loop" transmitter ("smoke ring in subsurface") secondary field generator will always be greater than the transmitter of the primary current loop. Thus, all the sounding points within the transmitter loop will be within the "secondary current loop". So, the calculation of apparent resistivity will be satisfactorily calculated. When the measurements are taken outside the transmitter loop the orientation of the $B$ field will vary with time and with distance from the side of the transmitter loop.

If the electrical currents just stay inside the transmitter loop ("on time" period), all apparent resistivity values outside the loop would be negative. But, the "smoke rings" 
a)

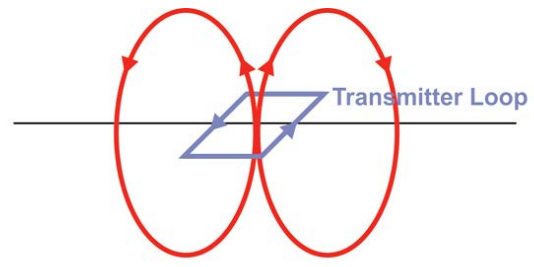

b)

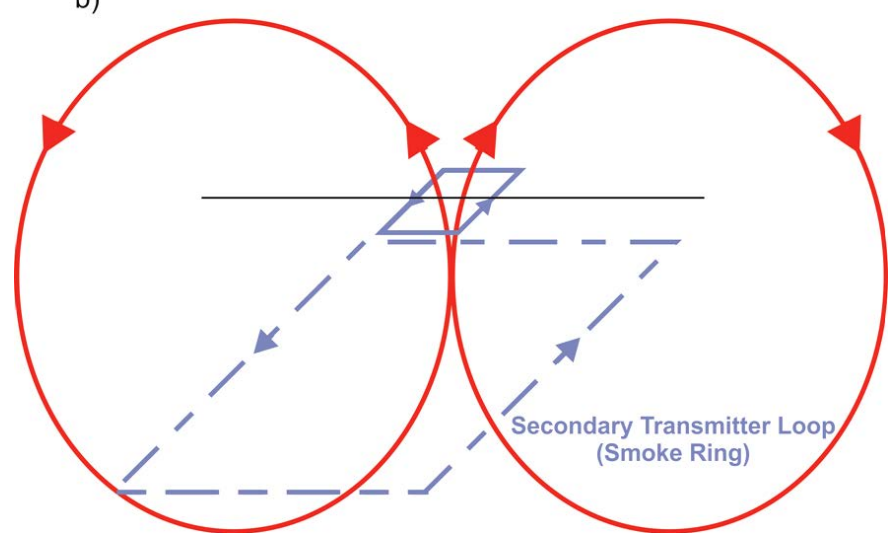

Figure 2. Schematic figure of how the secondary currents behave when compared to the primary current. In (a) the transmitter loop at the time the primary current passes through it, as well as the magnetic field $B$ associated with it. In (b), for a given time instant, the secondary currents in the subsurface with its associated magnetic field $B_{S}$.

(induced current) has increasingly diameter in relation to the passage of time, so the projection of "smoke rings" at the surface will cover an increased area. Thus, for longer times, greater distances will be inserted into this secondary current loop in subsurface. Then, in the shortest times the positions next to the transmitter loop have few negative apparent resistivity values, whereas for more distant positions from the loop side, the more negative values the curves have.

In Figure 3 this phenomenon is exemplify. For $t_{0}$, that is the "on time" period, the soundings inside the transmitter loop have positive values for all time positions, and the soundings outside the transmitter loop have negative apparent resistivities values for all time positions (Figure 3(a)). At time $t_{1}$ (Figure 3(b)) the electrical currents have already spread into subsurface to the positions of the receiver coil close to the transmitter loop and the soundings near the transmitter loop now have positive values of apparent resistivity, but the distant locations have negative values. As the currents will propagate in a "smoke rings" pattern bigger and deeper, more positions will have positive values.

However, in the case of real measurements usually a set of predetermined intervals are defined for the acquisition. What happens in practice is that for distant positions from the loop side, much of the resistivity curve is composed of negative values. In addition to negative values, the points just above the subsurface currents have the values of the $B_{Z}$ field practically zero, because the field lines are substantially horizontal. Thus, both the negative resistivity values as the values that are calculated from very small values $B_{Z}$ have a different behavior from those calculated inside the loop and/or positive. 


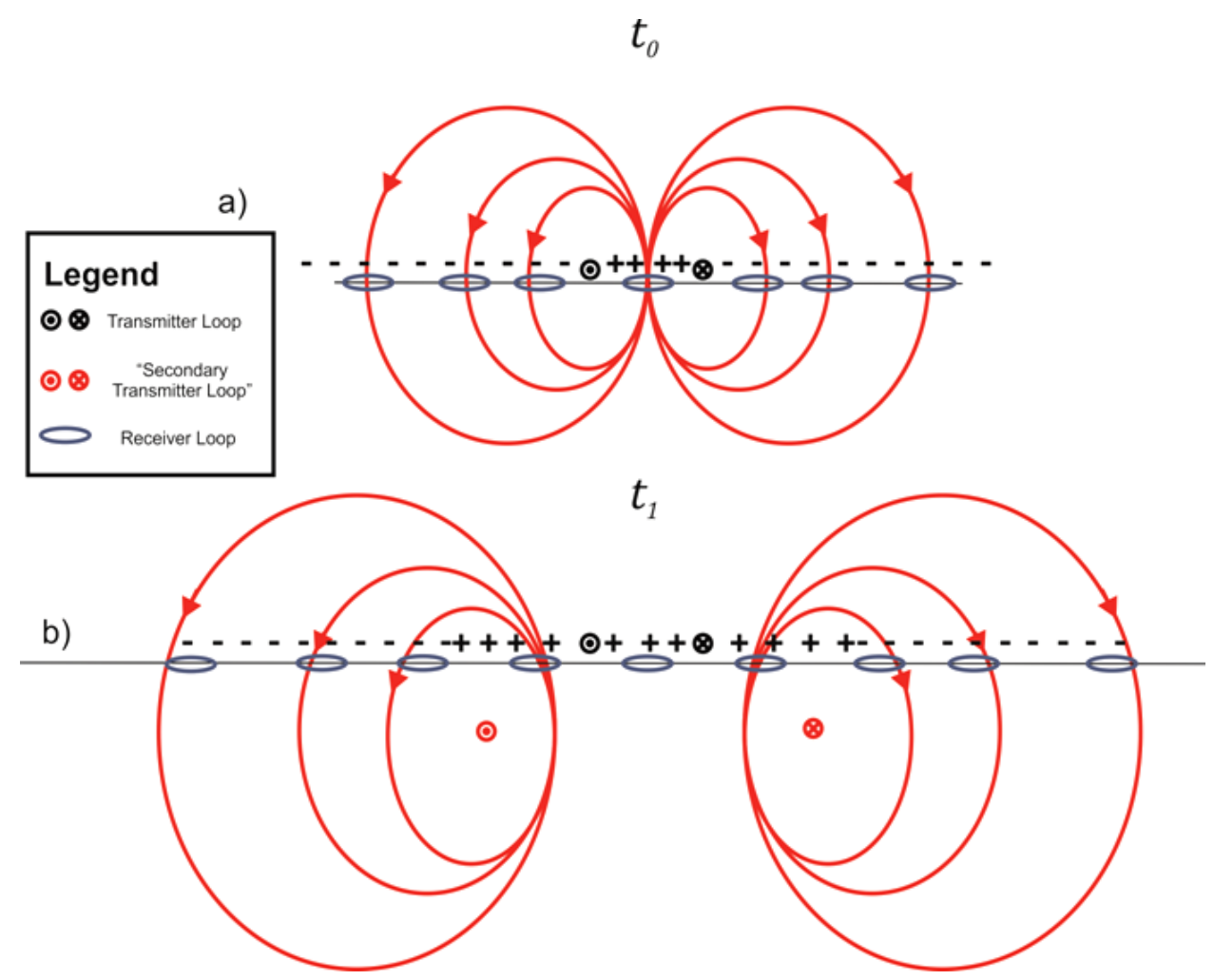

Figure 3. Schematic of the effect of orientation change of the fields $B$ and $B_{S}$ in the calculation of apparent resistivity inside and outside of the transmitter loop. (a) would be the "on time" period, $t_{0}$, and in (b), at time $t_{1}$, which already has the spread of secondary currents in the subsurface.

Direct modeling can be calculated for these points, but should take into account their different behavior.

To illustrate this effect is simulated three soundings inside and outside the transmitter loop with time intervals usually used in GEONICS TEM-57 equipment. The simulated geological medium is homogeneous with $10 \mathrm{Ohm} \cdot \mathrm{m}$ resistivity and a square loop of $200 \mathrm{~m} \times 200 \mathrm{~m}$. Figure 4 (a) is an example of direct calculation for a sounding point in the center of the transmitter loop (central loop array). In Figure 4(b) is presented a sounding point distant $150 \mathrm{~m}$ from the center of the transmitter loop, i.e., 50 meters outside from the side of the transmitter loop. In Figure 4(c) a survey at $500 \mathrm{~m}$ position from the center of the transmitter loop (400 $\mathrm{m}$ from the loop side). In Figure 4 the black dots are positive values of apparent resistivity and the red squares are the modulus of the negative apparent resistivity values. Figures clearly show the difference in the behavior of the apparent resistivity curves in three cases. The sounding in the center of the transmitter loop (Figure 4(a)) shows that the curve tends asymptotically to the real resistivity of the medium $(10 \mathrm{Ohm} \cdot \mathrm{m})$, showing that the apparent resistivity behavior does not have abrupt variations. In the case of sounding next to the side of the loop (Figure 4(b)), the beginning of the curve already has negative values and abrupt resistivity values. The peaks between positives and negatives values are the values of $\partial B_{Z} / \partial t$ very close to zero that are also not calculated accurately. Figure 4 (c) shows the 


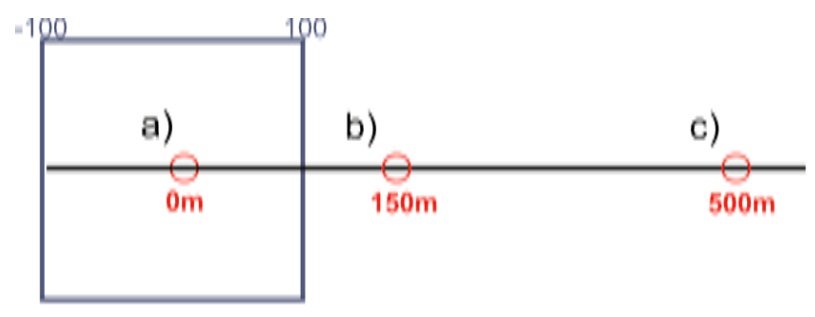

a)

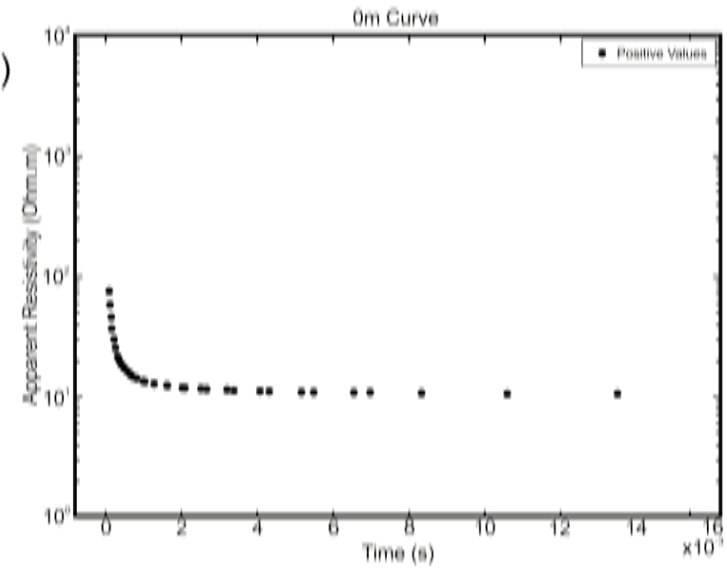

b)
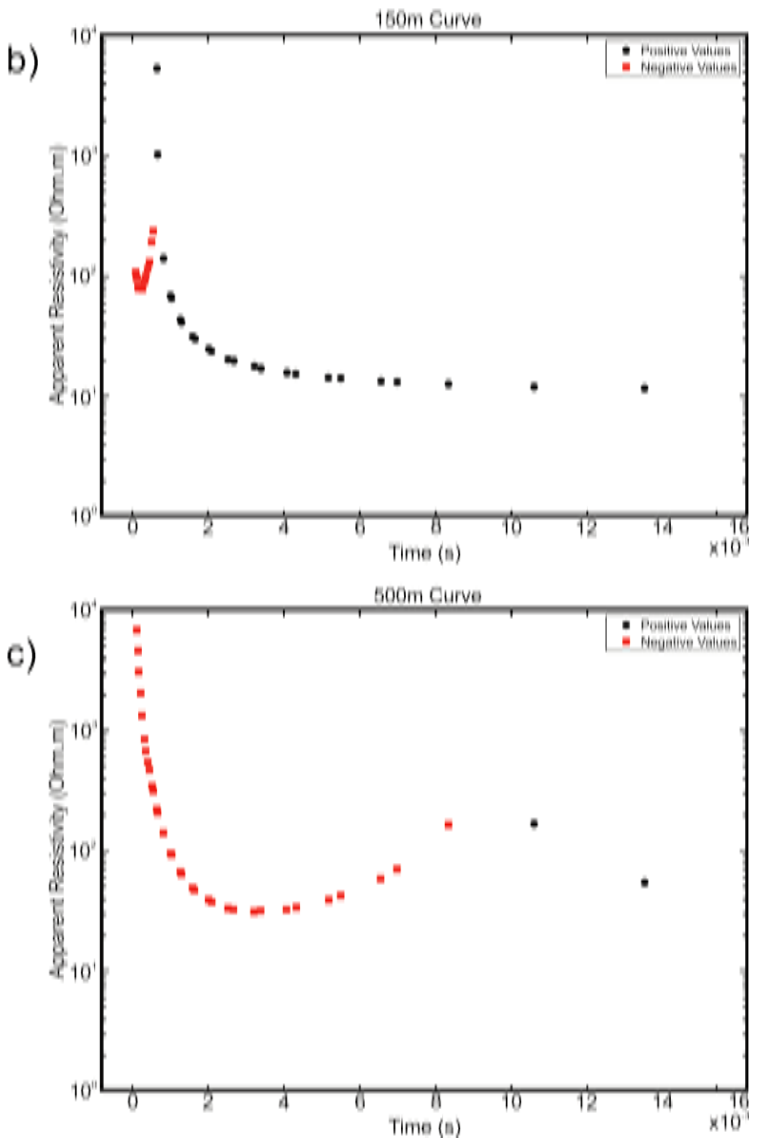

Figure 4. Soundings inside and outside of a transmitter loop with $200 \mathrm{~m} \times 200 \mathrm{~m}$. In (a) the sounding at position $0 \mathrm{~m}$ (center of the transmitter loop) in (b) the sounding at position $150 \mathrm{~m}$ (50 m outside the side of the transmitter loop) and (c) the sounding at $500 \mathrm{~m}$ (400 $\mathrm{m}$ outside the side of the transmitter loop). 
apparent resistivity curve for the survey at $500 \mathrm{~m}$ from the center of the array. It is possible to see that most of the curve is composed of negative values and even the two positive points are distant from the real resistivity $(10 \mathrm{Ohm} \cdot \mathrm{m})$, due to the fact the values of $\partial B_{Z} / \partial t$ are close to zero.

\section{Final Remarks}

Following this examination and some unsuccessfully synthetic tests with the $1 \mathrm{D}$ and 2D TEM inversion was concluded that the problem of negative values of apparent resistivity in TEM are still an open topic in geophysics. The negative values generate problems of stability and convergence in the inversion process, due to the fact that the formulas weren't designed to be used outside the transmitter loop. Even with the absolute values of apparent resistivity, sufficient stability was not obtained for a successfully inversion. This problem was described in the works of Campaña and Bortolozo [10], for the 1D and $2 \mathrm{D}$ cases respectively.

The solution for this question is not solved, once the major focus of recent studies was to determine a single formula for the calculation of the apparent resistivity in early and late times, without dealing with the question of soundings inside and outside the transmitter loop. The inversion using only the values of $\partial B_{Z} / \partial t$, despite possible, is not the final solution for this problem, due the subtle changes in the curve that may increase the ambiguity and lack of stability.

This article sought to highlight the problem of negative apparent resistivity values in TEM surveys and explain the function of the phenomena involved. The main objective is the discussion of this important and still open topic in the electromagnetic methods.

\section{Acknowledgements}

CAB thanks to Fundação de Amparo à Pesquisa do Estado de São Paulo (FAPESP) for the scholarship (2011/06404-0) and Conselho Nacional de Desenvolvimento Científico e Tecnológico (CNPq) for the Post Doctoral Scholarship (150230/2016-8). JDRC thanks to the Coordenação de Aperfeiçoamento de Pessoal de Nivel Superior (CAPES) for the scholarship. MACJ thanks to FAPESP for the scholarship (2012/07385-2). JLP thanks to FAPESP (2009/08466-3 and 2012/15338-4), CNPq-PVE (406653/2013-5) for the financial support to develop this research and CNPq (301692/2013-0) for research grant. FAPESP, CAPES and $\mathrm{CNPq}$ are Brazilian research agencies. Thanks to the Universidade de São Paulo for providing the infrastructure and support.

\section{References}

[1] Kaufman, A.A. and Keller, G.V. (1983). Frequency and Transient Soundings. Elsevier, Amsterdam.

[2] Oristaglio, M.L. and Hohmann, G.W. (1984) Diffusion of Electromagnetic Fields into a Two-Dimensional Earth: A Finite-Difference Approach. Geophysics, 49, 870-894. http://dx.doi.org/10.1190/1.1441733

[3] Nabighian, M.N. and Macnae, J.C. (1991) Time Domain Electromagnetic Prospecting Methods. In: Nabighian, M.N., Ed., Investigations in Geophysics No 3. Electromagnetic Methods 
in Applied Geophysics, Society of Exploration Geophysicists, Oklahoma, 427-514.

http://dx.doi.org/10.1190/1.9781560802686.ch6

[4] Nielsen, T.I. and Baumgartner, F. (2006) CR1Dmod: A Matlab Program to Model 1D Complex Resistivity Effects in Electrical and Electromagnetic Surveys. Computers \& Geosciences, 32, 1411-1419. http://dx.doi.org/10.1016/j.cageo.2006.01.001

[5] Guo, W., Li, X., Liu, Y. and Qi, Z. (2011) Apparent Resistivity Calculation Method of the Wide-Area, Multi-Component Transient Electromagnetic Field. Procedia Earth and Planetary Science, 3, 113-122. http://dx.doi.org/10.1016/j.proeps.2011.09.072

[6] Denghai, B., Meju, M.A., Jian, L., Lifeng, W. and Zhaohai, H. (2003) Numerical Calculation of All-Time Apparent Resistivity for Central Loop Transient Electromagnetic Method. Chinese Journal of Geophysics, 46, 998-1010. http://dx.doi.org/10.1002/cjg2.419

[7] Li, J., Zhu, Z., Feng, D., Xiao, J. and Peng, L. (2011) Calculation of All-Time Apparent Resistivity of Large Loop Transient Electromagnetic Method with Very Fast Simulated Annealing. Journal of Central South University of Technology, 18, 1235-1239.

http://dx.doi.org/10.1007/s11771-011-0827-y

[8] Campaña, J.D.R. (2015) Mapeamento de aquíferos sedimentares e fraturados na Bacia do Paraná por meio da técnica de caminhamento TDEM, usando Tx-fixo e Rx-móvel. MSc. Thesis, Universityof São Paulo, Brazil.

[9] Bortolozo, C.A. (2016) Inversão conjunta 1D e 2D de dados de Eletrorresistividadee TDEM aplicados em estudos de hidrogeologia na bacia do Paraná. São Paulo. PhD. Thesis, Universityof São Paulo, Brazil.

[10] Spies, B.R. and Parker, P.D. (1984) Limitations of Large-Loop Transient Electromagnetic Surveys in Conductive Terrains. Geophysics, 49, 902-912.

http://dx.doi.org/10.1190/1.1441735

\section{Submit or recommend next manuscript to SCIRP and we will provide best service for you:}

Accepting pre-submission inquiries through Email, Facebook, LinkedIn, Twitter, etc.

A wide selection of journals (inclusive of 9 subjects, more than 200 journals)

Providing 24-hour high-quality service

User-friendly online submission system

Fair and swift peer-review system

Efficient typesetting and proofreading procedure

Display of the result of downloads and visits, as well as the number of cited articles

Maximum dissemination of your research work

Submit your manuscript at: http://papersubmission.scirp.org/

Or contact ijg@scirp.org 